\title{
First-Principles Study of Electronic and Optical Properties of Two-Dimensional WSSe/BSe van der Waals Heterostructure with High Solar-to-Hydrogen Efficiency
}

\author{
Zhengyang Zhu ${ }^{1}$, Kai Ren ${ }^{2, *}\left(\mathbb{C}\right.$, Huabing Shu ${ }^{3} \mathbb{C}$, Zhen Cui ${ }^{4}$, Zhaoming Huang ${ }^{1, *}$, Jin $\mathrm{Yu}^{5}$ and Yujing $\mathrm{Xu}{ }^{2}$ \\ 1 School of Mechanical Engineering, Wanjiang University of Technology, Maanshan 243031, China; \\ Zhengyang_Zhu@126.com \\ 2 School of Mechanical and Electronic Engineering, Nanjing Forestry University, Nanjing 211189, China; \\ yujingxusun@gmail.com \\ 3 School of Science, Jiangsu University of Science and Technology, Zhenjiang 212001, China; \\ shuhuabing@just.edu.cn \\ 4 School of Automation and Information Engineering, Xi'an University of Technology, Xi'an 710048, China; \\ zcui@xaut.edu.cn \\ 5 School of Materials Science and Engineering, Southeast University, Nanjing 211189, China; yujin@seu.edu.cn \\ * Correspondence: kairen@njfu.edu.cn (K.R.); jimmymacy@163.com (Z.H.)
}

check for updates

Citation: Zhu, Z.; Ren, K.; Shu, H.; Cui, Z.; Huang, Z.; Yu, J.; Xu, Y. First-Principles Study of Electronic and Optical Properties of

Two-Dimensional WSSe/BSe van der Waals Heterostructure with High Solar-to-Hydrogen Efficiency. Catalysts 2021, 11, 991. https:// doi.org/10.3390/catal11080991

Academic Editor: Tzu-Hsuan Chiang

Received: 20 July 2021

Accepted: 17 August 2021

Published: 18 August 2021

Publisher's Note: MDPI stays neutral with regard to jurisdictional claims in published maps and institutional affiliations.

Copyright: (C) 2021 by the authors. Licensee MDPI, Basel, Switzerland. This article is an open access article distributed under the terms and conditions of the Creative Commons Attribution (CC BY) license (https:// creativecommons.org/licenses/by/ $4.0 /)$.

\begin{abstract}
In this paper, the optical and electronic properties of WSSe/BSe heterostructure are investigated by first-principles calculations. The most stable stacking pattern of the WSSe/BSe compounds is formed by van der Waals interaction with a thermal stability proved by ab initio molecular dynamics simulation. The WSSe/BSe heterostructure exhibits a type-I band alignment with direct bandgap of $2.151 \mathrm{eV}$, which can improve the effective recombination of photoexcited holes and electrons. Furthermore, the band alignment of the WSSe/BSe heterostructure can straddle the water redox potential at $\mathrm{pH} 0-8$, and it has a wide absorption range for visible light. In particular, the solar-to-hydrogen efficiency of the WSSe/BSe heterostructure is obtained at as high as $44.9 \%$ at $\mathrm{pH} 4$ and 5. All these investigations show that the WSSe/BSe heterostructure has potential application in photocatalysts to decompose water.
\end{abstract}

Keywords: two-dimensional materials; WSSe/BSe heterostructure; band alignment; optical property; first-principles calculations

\section{Introduction}

Since graphene was first prepared in 2004 [1], two-dimensional (2D) materials have attracted much attention due to their excellent electronic, optical, thermal and catalytic properties [2-6]. There are many kinds of elements in two-dimensional (2D) materials, including almost all of the elements [7]. One of most popular layered materials is transition metal dichalcogenides (TMDs), whose chemical composition is usually expressed by $M X_{2}$, in which the $M$ and $X$ represent a transition metal ( $\mathrm{Ti}, \mathrm{Ta}, \mathrm{W}$, etc.) and chalcogen ( $\mathrm{S}, \mathrm{Se}, \mathrm{Te}$, etc.), respectively [8,9]. The monolayer TMD materials could be extensively applied for the study of nano-devices [10], photocatalysts [11] and optoelectronics [12] because of their novel electronic properties and a direct bandgap of 1.1-2.0 eV [13]. In addition, considering their high flexibility and carrier transport properties in the atomic layer, TMDs are ideal for applications in flexible substrates and suitable for flexible and portable electronic devices $[9,14]$.

To extend the application range of 2D materials, recently, many new 2D materials have been predicted by the methods of global structure search and high throughput calculations $[15,16]$. For example, $\mathrm{B}_{2} \mathrm{P}_{6}$ was found to possess a Janus structure, which provides it with promising applications as a photocatalyst for water splitting and metal-ion batteries [17]. In addition, instead of a covalent bond [18], forming a 2D van der Waals (vdW) 
heterostructure is a common method to improve the properties of layered materials [19-21]. These weak vdW forces combine two different layered materials, which keep the heterostructure energetically stable $[22,23]$. The interface of a 2D heterostructure can create many interesting properties which enhance the electronic, optical and photocatalytic abilities [24-26]. Thus, a number of 2D materials have been built and used as p-n junctions [27], photocatalysts [28], field-effect transistors [29], etc. In detail, a vdW heterostructure with a sandwich structure based on $\mathrm{MoS}_{2}$ - graphene-WSe 2 was prepared by the mechanical exfoliation method possessing a wide absorption spectrum, which demonstrates that the heterostructure has a specific detectivity of up to $10^{11}$ Jones [30]. The graphene/ $\mathrm{MoS}_{2}$ heterostructure was formed by chemical vapor deposition (CVD), and the heterostructure was used in high-performance devices and circuits, with $\mathrm{MoS}_{2}$ and graphene acting as the transistor channel and contact electrodes, respectively. Importantly, the novel electronic and optical properties of 2D heterostructures also can been tuned by electric fields [31], external biaxial strain [32], defects [33], etc. The above reports show that vdW heterostructures can be used as candidates for high-performance nano-electronic and optoelectronic devices.

Recently, the TMD materials with a Janus structure have widely been investigated, since the MoSSe was grown using a synthetic strategy with vertical dipoles [34]. Then, the Raman vibrational modes and electronic structures of the Janus MoSSe monolayer were calculated by means of density functional theory (DFT) and compared with the corresponding experimental results. It was found that Janus monolayers have high activity of hydrogen evolution on the base surface. Density functional calculation shows that the activity comes from the mutual effect of structural strain and inherent defects of Janus MoSSe [35]. Similarly, the Janus WSSe monolayer also has superior carrier mobility of about $600 \mathrm{~cm}^{2} \cdot \mathrm{V}^{-1} \cdot \mathrm{s}^{-1}$, which is more advantageous than conventional TMD monolayers and other Janus TMD materials [36]. Considering the pronounced electronic and optical properties of the WSSe monolayer, it has been constructed as a heterostructure with MoSSe and possesses intrinsic many-body effects. Both horizontal and vertical WSSe/MoSSe heterostructures have type-II band alignment [37]. More recently, a boron selenide (BSe) monolayer was studied as a semiconductor with an indirect bandgap [38]. Furthermore, BSe-based heterostructures are also addressed by g-GaN/BSe [23], ZnO/BSe [24], blue phosphorene/BSe [39], etc., which shows the promising applications for BSe-based heterostructures in electronics, spintronics, and optoelectronics.

In this paper, based on DFT, some interesting properties of the WSSe and BSe monolayers and the WSSe/BSe heterostructure are addressed. The WSSe/BSe heterostructure is decided by the stacking structure with lowest binding energy, and the thermal stability of the WSSe/BSe heterostructure is examined. Then, the interfacial properties of the WSSe/BSe heterostructure are investigated according to the potential drop and charge density difference. The optical absorption property and optical conversion efficiency of the WSSe/BSe heterostructure are also studied. Our research can provide effective guidance for the application of such vdW heterostructures in future nano-electronic and optoelectronic devices.

\section{Computing Method}

Based on density functional theory (DFT), first-principles calculations were conducted using the Vienna ab initio simulation package (VASP) [40,41]. The projector-augmented wave method with energy cutoff of $550 \mathrm{eV}$ was considered. The generalized gradient approximation (GGA) [42] of Perdew-Burke-Ernzerhof functional (PBE) was used to explain the electron exchange and correlation potential [43-45]. For the accurate electronic and optical properties, the Heyd-Scuseria-Ernzerhof (HSE06) method was utilized [46]. The Grimme correction method (DFT-D3) was explored to describe the weak dispersion for the vdW correction [47]. In the first Brillouin zone, $11 \times 11 \times 1 k$-point mesh was employed. The vacuum thickness of $25 \AA$ was considered to prevent the interaction of nearby layers. In addition, the convergence criteria of total energy and the Hellmann-Feynman forces for 
the atoms of the system investigated in this work were $1 \times 10^{-5} \mathrm{eV}$ and $0.01 \mathrm{eV} \cdot \AA^{-1}$, respectively. Furthermore, we built the model of monolayered WSSe, BSe and the WSSe/BSe heterostructure with the initial lattice constants of $3.23,3.25$ and $3.24 \AA$, respectively $[48,49]$. Then, we relaxed all of the systems and obtained the appropriate lattice constants.

\section{Results and Discussion}

First, the structures were optimized for the pristine WSSe and BSe monolayers shown in Figure 1a,c, respectively, at 3.269 and $3.245 \AA$ for lattice constants. The bond lengths of the W-S, W-Se and B-Se in the monolayered WSSe and BSe were obtained as 2.428, 2.542 and $2.099 \AA$, respectively. Furthermore, using HSE06 calculations and the PBE method, the band structures of both monolayered materials are calculated in Figure 1a,b, and one can find that the WSSe has a direct bandgap of $2.077 \mathrm{eV}$ (HSE results) with the conduction band minimum (CBM) and the valence band maximum (VBM) located at the $\mathrm{K}$ points, while the BSe possesses an indirect bandgap of $3.466 \mathrm{eV}$. The CBM of the BSe appears at the point $\mathrm{M}$, while the VBM is located at the $\Gamma$ point. In addition, all these findings are consistent with previous reports $[50,51]$, which proves that our computational methods are accurate and reliable.

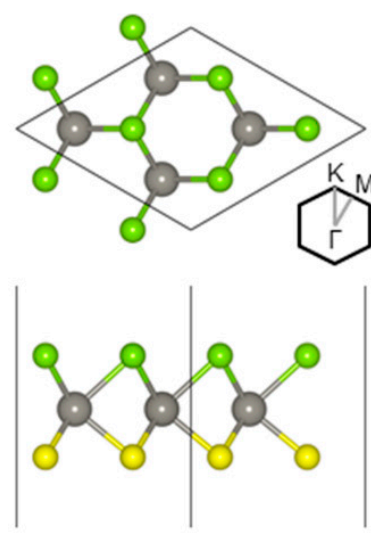

(a)

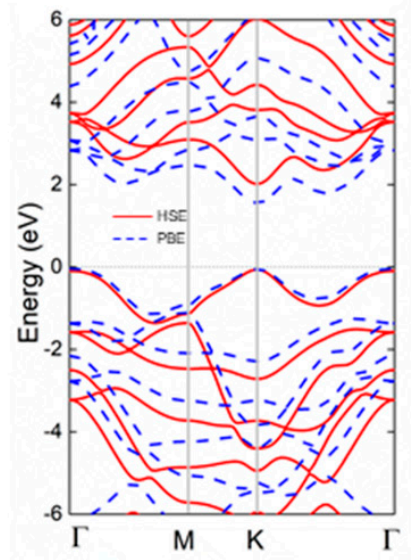

(b)
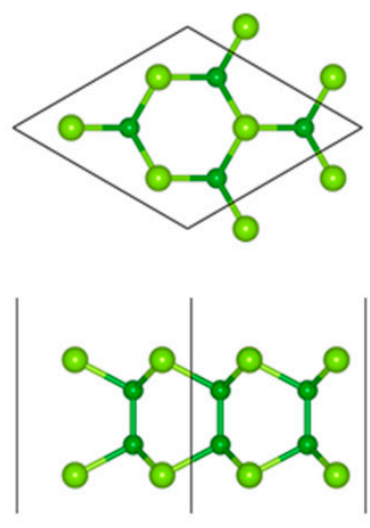

(c)

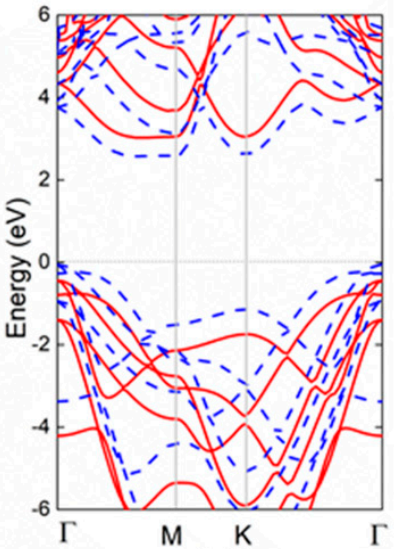

(d)

Figure 1. The crystal structure of the (a) WSSe and (c) BSe monolayers; the band structure of the (b) WSSe and (d) BSe monolayers calculated by HSE06 (red line) and PBE (blue marks) methods, respectively. The gray, grey, green and yellow marks represent Se, W, B and S atoms, respectively. The Fermi level is set by 0 pointed by the gray dashed line.

Next, we constructed the heterostructure composed by WSSe and BSe monolayers. As the lattice parameters of the WSSe and BSe monolayers shows very small lattice mismatch (about $0.74 \%$ ), they are suitable to be designed as a heterostructure, which can induce a precise stacking configuration. Here, we considered 12 possible highly symmetric stacking patterns for the WSSe/BSe heterostructure, as presented in Figure 2. $\gamma-1, \gamma-2, \gamma-3, \gamma-4, \gamma-5$ and $\gamma-6$ stacking are similar with $\gamma-7, \gamma-8, \gamma-9, \gamma-10, \gamma-11$ and $\gamma-12$ stacking, respectively, by exchanging the site of the $S$ and Se atoms in the WSSe layer. For $\gamma-1, W$ and Se (or S) atoms are put on top of B atoms and Se atoms, respectively. The $\gamma-2$ style is obtained by changing the positions of $B$ and Se atoms from the $\gamma-1$ configuration. Meanwhile, for $\gamma-3$, $\mathrm{W}$ atoms are positioned on top of the hexagonal center of the BSe, and Se (or S) atoms are located on top of Se atoms in BSe layer. $\gamma-4$ can be constructed by locating the Se (or S) atoms on the top of the hexagonal center of BSe and W atoms on the top of the Se atoms in the BSe layer. For the $\gamma-5$ style, Se (or $S$ ) atoms are positioned on the top of the hexagonal center of BSe, and $W$ atoms are located on the top of $B$ atoms. $\gamma-6$ is built by changing the positions of $\mathrm{W}$ and Se (or S) fromm $\gamma-5$. 


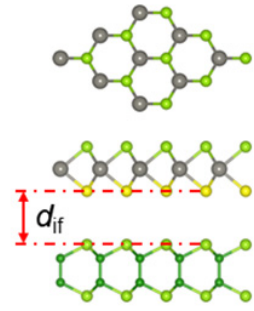

(a)

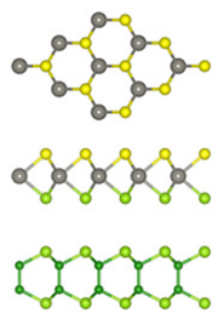

(g)

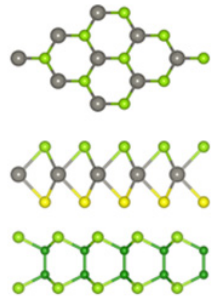

(b)
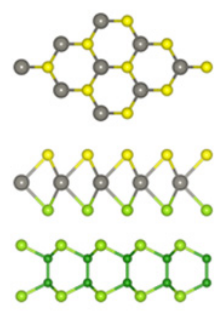

(h)

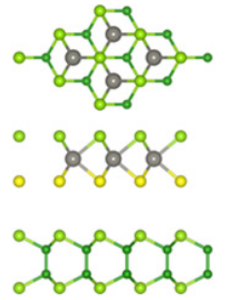

(c)

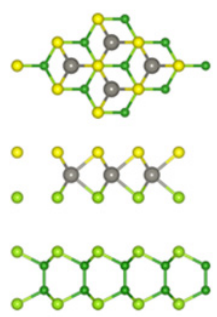

(i)

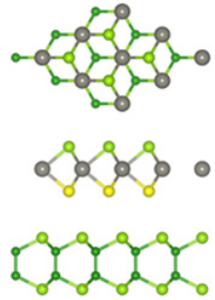

(d)

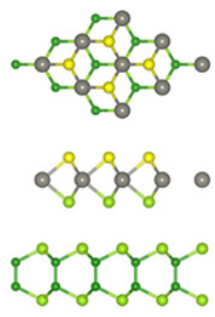

(j)

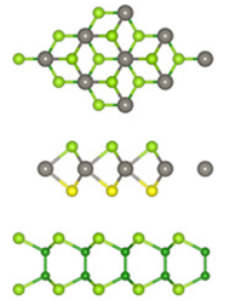

(e)
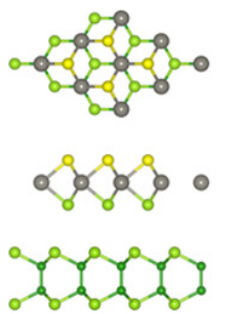

(k)

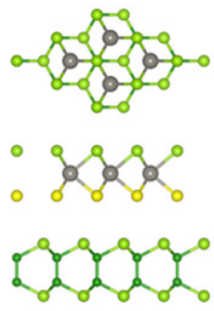

(f)

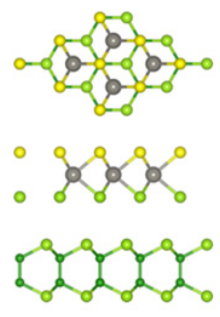

(I)

Figure 2. The different stacking patterns of WSSe/BSe heterostructure: (a) $\gamma-1 ;$ (b) $\gamma-2$; (c) $\gamma-3$; (d) $\gamma-4$; (e) $\gamma-5$; (f) $\gamma-6$; (g) $\gamma-7$; (h) $\gamma-8$; (i) $\gamma-9 ;$ (j) $\gamma-10 ;(\mathbf{k}) \gamma-11 ;$ (l) $\gamma-12$

For all those representative highly symmetric stacking patterns, we judged the most stable one by calculating the binding energy $\left(E_{\mathrm{b}}\right)$ as follows:

$$
E_{\mathrm{b}}=\left(E_{\mathrm{WSSe}-\mathrm{BSe}}-E_{\mathrm{WSSe}}-E_{\mathrm{BSe}}\right) / A
$$

where $E_{\text {WSSe/BSe }}, E_{\text {WSSe }}$ and $E_{\mathrm{BSe}}$ are the energy of the WSSe/BSe heterostructure and the original WSSe and BSe monolayers, respectively. $A$ is the area of the cell. The obtained $E_{\mathrm{b}}$ and interface distance $\left(d_{\text {if }}\right)$ are addressed in Table 1 . The $\gamma-10$ stacking patterns possesses the lowest binding energy of $-25.444 \mathrm{meV} / \AA^{-2}$, which is smaller than that in the vdW bonding in weak interlayer interactions in graphites of about $-18 \mathrm{meV} / \AA^{-2}$, demonstrating that the WSSe/BSe heterostructure is formed by vdW interactions [52]. In addition, the bond lengths of the W-S, W-Se and B-Se in the monolayered WSSe and BSe only show a slight change in the heterostructure, which further explains the weak vdW interactions between the interface of the heterostructure. Therefore, all of the following investigations are focused on the $\gamma-10$ stacking configuration.

Table 1. The binding energy $\left(E_{\mathrm{b}}, \mathrm{meV} / \AA^{-2}\right)$, interface distance $\left(d_{\mathrm{if}}, \AA\right)$, and bond length $(L, \AA)$ of $\mathrm{W}-\mathrm{S}, \mathrm{W}-\mathrm{Se}$ and B-Se in WSSe and BSe, respectively.

\begin{tabular}{cccccc}
\hline Configuration & $\boldsymbol{E}_{\mathbf{b}}$ & $\boldsymbol{d}_{\mathbf{i f}}$ & $\boldsymbol{L}_{\mathbf{W}-\mathbf{S}}$ & $\boldsymbol{L}_{\mathbf{W}-\mathbf{S e}}$ & $\boldsymbol{L}_{\mathbf{B}-\mathbf{S e}}$ \\
\hline$\gamma-1$ & -18.444 & 3.783 & 2.422 & 2.534 & 2.101 \\
$\gamma-2$ & -24.889 & 3.283 & 2.421 & 2.537 & 2.101 \\
$\gamma-3$ & -18.556 & 3.774 & 2.422 & 2.538 & 2.101 \\
$\gamma-4$ & -25.000 & 3.279 & 2.421 & 2.538 & 2.100 \\
$\gamma-5$ & -24.000 & 3.355 & 2.421 & 2.538 & 2.101 \\
$\gamma-6$ & -24.444 & 3.332 & 2.421 & 2.538 & 2.101 \\
$\gamma-7$ & -18.889 & 3.884 & 2.423 & 2.537 & 2.101 \\
$\gamma-8$ & -25.333 & 3.397 & 2.423 & 2.537 & 2.100 \\
$\gamma-9$ & -19.000 & 3.873 & 2.423 & 2.537 & 2.101 \\
$\gamma-10$ & -25.444 & 3.397 & 2.423 & 2.536 & 2.101 \\
$\gamma-11$ & -23.778 & 3.478 & 2.423 & 2.536 & 2.101 \\
$\gamma-12$ & -24.223 & 3.486 & 2.423 & 2.536 & 2.101 \\
\hline
\end{tabular}

Then, we evaluated the thermal stability of the WSSe/BSe vdW heterostructure by ab initio molecular dynamics (AIMD) simulation with a Nosé-Hoover heat bath scheme [53]. In this simulation, due to the lattice translation constraint, we built a $6 \times 6 \times 1$ supercell for 
the WSSe/BSe vdW heterostructure including 252 atoms. After completing the simulation calculations, as shown in Figure 3a, the WSSe/BSe vdW heterostructures were still integral after 5 ps at $300 \mathrm{~K}$, demonstrating their pronounced thermal stability at room temperature. Moreover, with the simulation step, the change in the total energy of the WSSe/BSe $\mathrm{vdW}$ heterostructure and the fluctuation in temperature are shown in Figure $3 \mathrm{~b}$, which demonstrates a convergence with the simulation. It is worth noting that the $\gamma-8 \mathrm{WSSe} / \mathrm{BSe}$ vdW heterostructure shows little difference in the binding energy (about $0.111 \mathrm{meV} / \AA^{-2}$ ) compared with the $\gamma-10$ WSSe/BSe vdW heterostructure, and so the thermal stability of the $\gamma-8$ WSSe/BSe vdW heterostructure was also addressed in Supplementary Figure S1, in the Supporting Information, which suggests that the $\gamma-8$ WSSe/BSe vdW heterostructure possesses a thermal stability at room temperature.
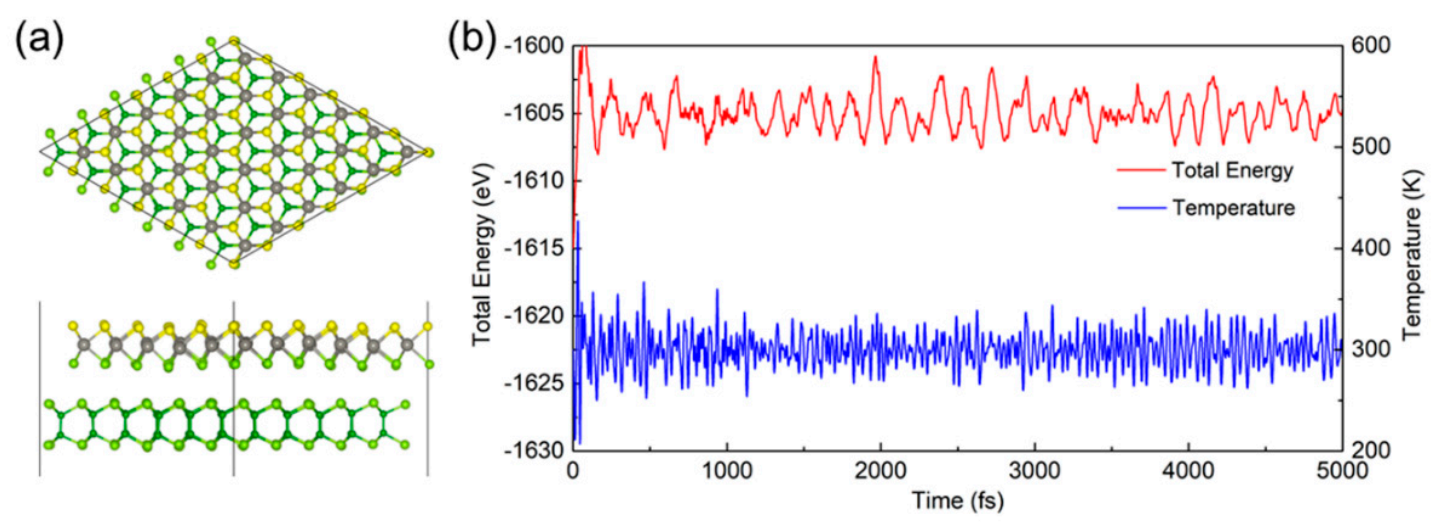

Figure 3. (a) The AIMD snapshots for the WSSe/BSe vdW heterostructure; (b) the total energy and temperature fluctuations during AIMD simulations for the WSSe/BSe heterostructure.

We also calculated the projected band structure for the WSSe/BSe vdW heterostructure, shown in Figure 4a, obtained by using HSE06 calculations. We found that the WSSe/BSe $\mathrm{vdW}$ heterostructure has a direct bandgap of $2.151 \mathrm{eV}$, which means that it is a semiconductor. It is interesting that both the VBM and CBM of the heterostructure are donated from the WSSe layer, exhibiting a type-I band alignment, as shown in Figure 4a. Furthermore, we calculated the projected density of states of the WSSe/BSe vdW heterostructure shown in Figure $4 \mathrm{~b}$, which can further prove the type-I band alignment in the WSSe/BSe vdW heterostructure. Previous studies have shown that a potential photocatalyst should possess decent electronic characteristics, which can be used to decompose water [54]. Firstly, the band gap should be between 1.23 and $3.0 \mathrm{eV}$. Secondly, the band edge positions of the photocatalyst should meet the water redox potentials, which means that the potential of the VBM should be lower than $-5.67 \mathrm{eV}$ and the potential of the CBM of materials should be higher than $-4.44 \mathrm{eV}$ [39]. The calculated bandgap (2.151 eV) of the WSSe/BSe vdW heterostructure reveals the suitable bandgap value as a photocatalyst to decompose water [12], and the band edge positions of the monolayered WSSe, BSe and WSSe/BSe vdW heterostructure are calculated in Figure 4c, showing that the band alignments of these layered materials all possess decent potential for the oxidation and reduction reactions to decompose water at $\mathrm{pH} 0$. 

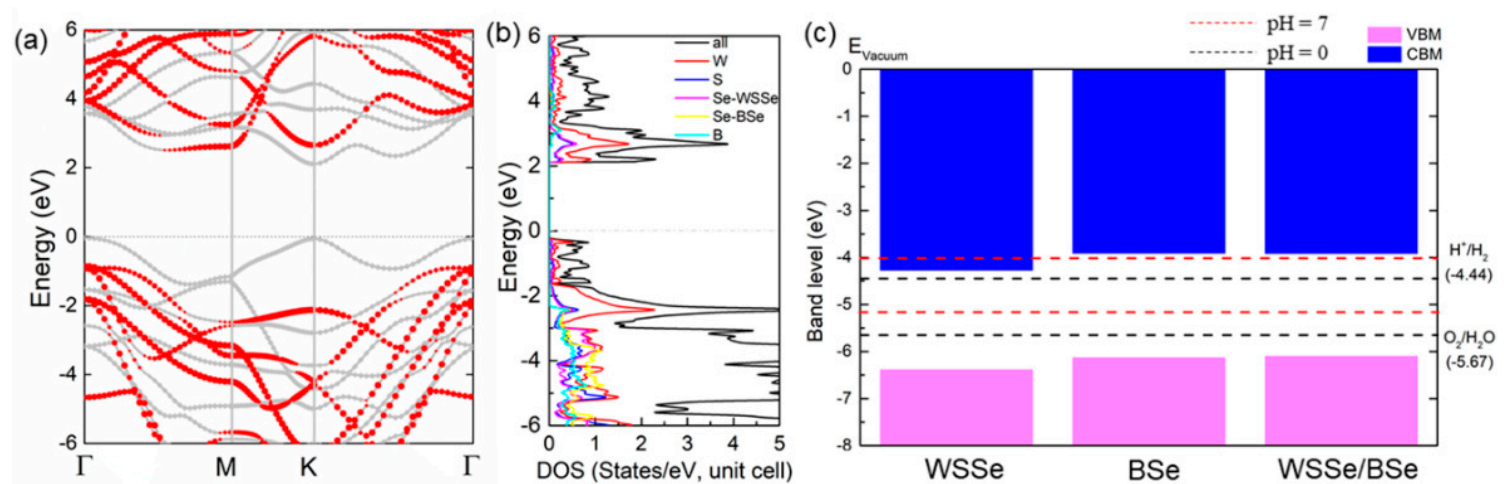

Figure 4. (a) The projected band structure and (b) the projected density of states of the WSSe/BSe vdW heterostructure; (c) the band edge positions of the WSSe, BSe and the WSSe/BSe vdW heterostructure, and the potentials of redox at $\mathrm{pH} 0$ and 7 to decompose water are also shown.

Considering the influence of the $\mathrm{pH}$ value, the reduction and oxidation potentials are obtained from the value of the $\mathrm{pH}$ as: $E_{\text {reduction }}=-4.44 \mathrm{eV}+\mathrm{pH} \times 0.059 \mathrm{eV}$, and $E_{\text {oxidation }}=-5.67 \mathrm{eV}+\mathrm{pH} \times 0.059 \mathrm{eV}$, respectively [55]. As shown in Figure 4c, the band alignment of the WSSe/BSe vdW heterostructure still possesses decent band edge position energy level for the water redox potentials at $\mathrm{pH}$ 7, but the WSSe monolayer is not suitable for the photocatalyst for the hydrogen evolution reaction (HER). Furthermore, we investigated the potentials of the oxygen evolution reactions (OER) and HER to decompose water at different $\mathrm{pH}$ compared with the band edge energy of the WSSe/BSe vdW heterostructure in Figure 5, showing that the WSSe/BSe vdW heterostructure still can induce the OER and HER at $\mathrm{pH} 0$ to 8 to decompose the water.

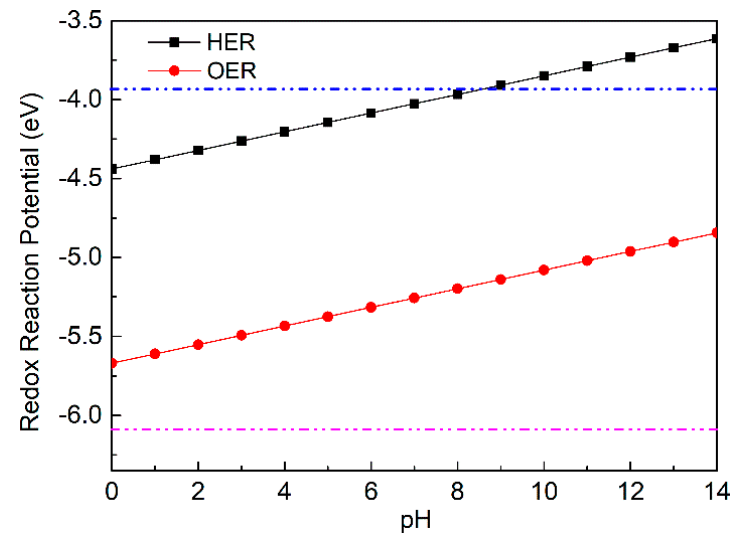

Figure 5. The potentials of the OER and HER of the water splitting as a function of the $\mathrm{pH}$ value with respect to the band edge positions of the WSSe/BSe vdW heterostructure.

As for the interfacial properties of the vdW heterostructure in this work, the charge density differences $(\Delta \rho)$ were investigated by:

$$
\Delta \rho=\rho_{\mathrm{WSSe}} / \mathrm{BSe}-\rho_{\mathrm{WSSe}}-\rho_{\mathrm{BSe}}
$$

where $\rho_{\mathrm{WSS} e / \mathrm{BSe}}, \rho_{\mathrm{WSSe}}$ and $\rho_{\mathrm{BSe}}$ mean the charge density difference of the WSSe/BSe heterostructure and the isolated WSSe and BSe layers, respectively. As shown as Figure 6a, the cyan regions represent the negative values of chargers, and the yellow regions represent the positive values, which shows that the charge redistribution is mainly around the interface of the WSSe/BSe vdW heterostructure. In addition, the Bader charge analysis method $[56,57]$ was used to calculate the charge transfer between the WSSe and BSe layers. We found that there are only 0.0047 transferred electrons from the BSe layer to WSSe 
layer. Moreover, the electrostatic potential drop across the interface of the WSSe/BSe vdW heterostructure is calculated in Figure 6b, which indicates that the WSSe layers present a slightly lower potential than the BSe layers in their corresponding heterostructure. This contributes to the formation of a built-in electric field, which is also an important factor for carrier migration.
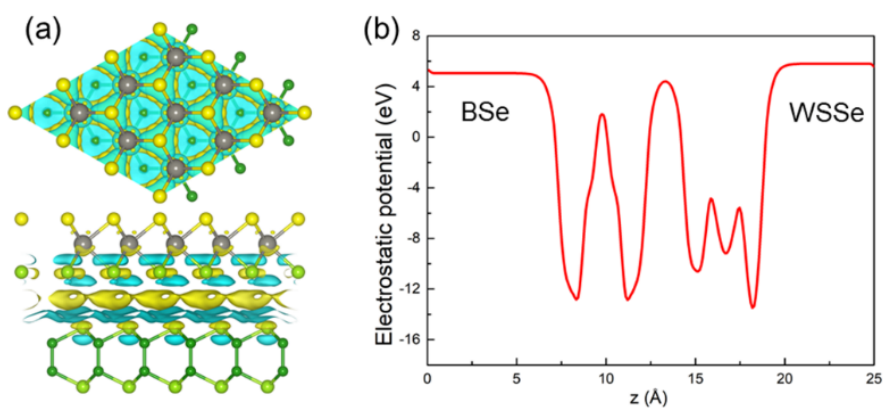

Figure 6. (a) The charge density and the (b) potential drop of the WSSe/BSe vdW heterostructure; the cyan and yellow regions indicate the loss and gain of the electron, respectively; the isosurface of charge difference was set to $10^{-4}|e|$.

We further investigated the optical properties of the WSSe/BSe vdW heterostructure. The solar-to-hydrogen (STH) efficiency is critical, which is also influenced by the pH values. The efficiency of the STH $\left(\eta_{\mathrm{STH}}\right)$ is decided by:

$$
\eta_{\mathrm{STH}}=\eta_{\mathrm{abs}} \times \eta_{\mathrm{cu}}
$$

where $\eta_{\mathrm{abs}}$ and $\eta_{\mathrm{cu}}$ are called the efficiency of light absorption and carrier utilization, respectively. Additionally, the $\eta_{\text {abs }}$ is calculated as:

$$
\eta_{\mathrm{abs}}=\frac{\int_{E_{\mathrm{g}}}^{\infty} P(h \omega) \mathrm{d}(h \omega)}{\int_{0}^{\infty} P(h \omega) \mathrm{d}(h \omega)}
$$

in which the $P(h \omega)$ is demonstrated for the solar energy flux of AM1.5G by the energy of $h \omega$ for phonon, and where $E_{\mathrm{g}}$ is used for the bandgap of the heterostructure. The $\eta_{\mathrm{cu}}$ is obtained by:

$$
\eta_{\mathrm{cu}}=\frac{\Delta G \int_{E}^{\infty} \frac{P(h \omega)}{h \omega} \mathrm{d}(h \omega)}{\int_{E_{\mathrm{g}}}^{\infty} P(h \omega) \mathrm{d}(h \omega)}
$$

where the potential difference is described by $\Delta G$, which explains water splitting of $1.23 \mathrm{eV}$. $E$ is the energy of the phonon in water splitting, calculated by:

$$
E=\left\{\begin{array}{r}
E_{g},\left(\chi\left(\mathrm{H}_{2}\right) \geq 0.2, \chi\left(\mathrm{O}_{2}\right) \geq 0.6\right) \\
E_{g}+0.2-\chi\left(\mathrm{H}_{2}\right),\left(\chi\left(\mathrm{H}_{2}\right)<0.2, \chi\left(\mathrm{O}_{2}\right) \geq 0.6\right) \\
E_{g}+0.6-\chi\left(\mathrm{O}_{2}\right),\left(\chi\left(\mathrm{H}_{2}\right) \geq 0.2, \chi\left(\mathrm{O}_{2}\right)<0.6\right) \\
E_{g}+0.8-\chi\left(\mathrm{H}_{2}\right)-\chi\left(\mathrm{O}_{2}\right),\left(\chi\left(\mathrm{H}_{2}\right)<0.2, \chi\left(\mathrm{O}_{2}\right)<0.6\right)
\end{array}\right.
$$

where the overpotential of HER and OER are addressed by $\chi\left(\mathrm{H}_{2}\right)$ and $\chi\left(\mathrm{O}_{2}\right)$, respectively. Generally, 0.2 and $0.6 \mathrm{eV}$ are suggested to be the required overpotentials for the HER and OER, as reported by a previous experimental study [58]. We calculated the $\eta_{\mathrm{STH}}$ of the WSSe/BSe vdW heterostructure at different $\mathrm{pH}$ values, as shown in Figure 7a. The WSSe/BSe vdW heterostructure possesses a novel solar-to-hydrogen efficiency at $\mathrm{pH} 4$ or 5 , of about $44.9 \%$, which is higher than that for other 2D heterostructures, such as $\mathrm{PtS}_{2}$ /arsenene [59], arsenene/GaS and arsenene/GaSe [22]. In addition, the WSSe/BSe $\mathrm{vdW}$ heterostructure also possesses decent solar-to-hydrogen efficiency, of about $21.4 \%$ and $25.4 \%$ at $\mathrm{pH} 0-3$ and $\mathrm{pH} 6-8$, respectively. 

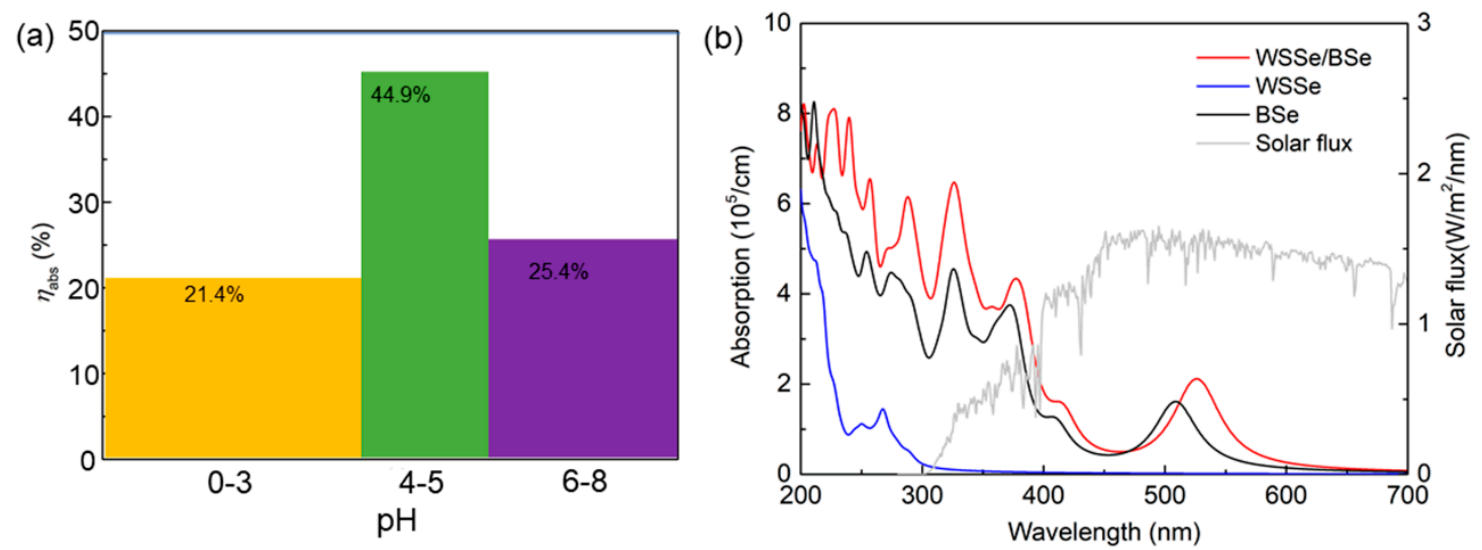

Figure 7. The calculated (a) STH efficiency at different $\mathrm{pH}$ values and (b) optical absorption of the WSSe/BSe vdW heterostructure.

The WSSe/BSe vdW heterostructure's ability to absorb light is also obtained by:

$$
\alpha(\omega)=\frac{\sqrt{2} \omega}{c}\left\{\left[\varepsilon_{1}^{2}(\omega)+\varepsilon_{2}^{2}(\omega)\right]^{1 / 2}-\varepsilon_{1}(\omega)\right\}^{1 / 2}
$$

where $\varepsilon_{1}(\omega)$ represents the real parts of the dielectric constant, and $\varepsilon_{2}(\omega)$ represents the imaginary parts, as shown as Figure 7b. The peaks of optical absorption for the WSSe, BSe and the WSSe/BSe vdW heterostructure are $2.11 \times 10^{5}, 1.44 \times 10^{5}$ and $1.61 \times 10^{5} \mathrm{~cm}^{-1}$, located at the wavelengths of 523, 511 and $266 \mathrm{~nm}$, respectively, which means that the WSSe monolayer and the WSSe/BSe vdW heterostructure possess novel optical absorption properties in the visible-light region. However, the WSSe does not have decent redox potential of HER for water splitting; thus, the WSSe/BSe vdW heterostructure is more suitable for use as a photocatalyst for water splitting.

\section{Conclusions}

In conclusion, we have systematically investigated some interesting features of the 2D WSSe, BSe and WSSe/BSe heterostructure by means of first-principles calculation. Our results demonstrate that the WSSe/BSe heterostructure can be constructed by vdW forces with thermal stability. Furthermore, the WSSe/BSe vdW heterostructure preserves the semiconductor feature of the original layered materials with a direct bandgap of $2.151 \mathrm{eV}$, and exhibits type-I band alignment. Furthermore, the WSSe, BSe and WSSe/BSe vdW heterostructure possess decent band edge positions to induce the HER and OER for water splitting at $\mathrm{pH} 0$, while the WSSe/BSe vdW heterostructure still has excellent potential for redox reactions, decomposing the water at a $\mathrm{pH}$ value of $0-8$. Furthermore, the WSSe/BSe vdW heterostructure can exhibit a novel STH efficiency, of up to about $44.9 \%$, and a broader visible-light absorption range. All these findings demonstrate that the WSSe/BSe vdW heterostructure could be used as a potential photocatalyst to decompose water, and is a potential candidate for use in high-performance nano-electronic and optoelectronic devices.

Supplementary Materials: The following are available online at https: / www.mdpi.com/article / 10.3390/catal11080991/s1, Figure S1: The AIMD snapshots for WSSe/BSe vdW heterostructure with $\gamma-8$ stacking style; the total energy and temperature fluctuations during AIMD simulations for WSSe/BSe heterostructure with $\gamma-8$ stacking style.

Author Contributions: Conceptualization, Z.Z. and K.R.; methodology, K.R.; software, J.Y.; validation, Z.Z. and K.R.; formal analysis, K.R.; investigation, Y.X.; resources, K.R.; data curation, K.R.; writing-original draft preparation, Z.Z.; writing—review and editing, H.S.; visualization, Z.C.; supervision, K.R.; project administration, Z.Z.; funding acquisition, Z.H. All authors have read and agreed to the published version of the manuscript. 
Funding: This work was supported by the Open Fund Project of Maanshan Engineering Technology Research Center of Advanced Design for Automotive Stamping Dies (Grant number: QMSG202003 and QMSG202105), the National Natural Science Foundation of Natural Science Basic Research Plan in Shaanxi Province of China (No.2021JM-345), China Postdoctoral Science Foundation (No.2020M683684XB).

Conflicts of Interest: There are no conflict to declare.

\section{References}

1. Geim, A.K.; Novoselov, K.S. The rise of graphene. Nat. Mater. 2007, 6, 183-191. [CrossRef] [PubMed]

2. Miro, P.; Audiffred, M.; Heine, T. An atlas of two-dimensional materials. Chem. Soc. Rev. 2014, 43, 6537-6554. [CrossRef] [PubMed]

3. Li, L.; Han, W.; Pi, L.; Niu, P.; Han, J.; Wang, C.; Su, B.; Li, H.; Xiong, J.; Bando, Y. Emerging in-plane anisotropic two-dimensional materials. Info Mat. 2019, 1, 54-73. [CrossRef]

4. Sun, M.; Luo, Y.; Yan, Y.; Schwingenschlögl, U. Ultrahigh Carrier Mobility in the Two-Dimensional Semiconductors $\mathrm{B}_{8} \mathrm{Si}_{4}, \mathrm{~B}_{8} \mathrm{Ge}_{4}$, and $\mathrm{B}_{8} \mathrm{Sn}_{4}$. Chem. Mater. 2021. [CrossRef]

5. Ren, K.; Liu, X.; Chen, S.; Cheng, Y.; Tang, W.; Zhang, G. Remarkable reduction of interfacial thermal resistance in nanophononic heterostructures. Adv. Funct. Mater. 2020, 30, 2004003. [CrossRef]

6. Zheng, Z.; Ren, K.; Huang, Z.; Zhu, Z.; Wang, K.; Shen, Z.; Yu, J. Remarkably improved curie temperature for two-dimensional $\mathrm{CrI}_{3}$ by gas molecular adsorption: A dft study. Semicond. Sci. Technol. 2021, 36, 075015. [CrossRef]

7. Butler, S.Z.; Hollen, S.M.; Cao, L.; Cui, Y.; Gupta, J.A.; Gutiérrez, H.R.; Heinz, T.F.; Hong, S.S.; Huang, J.; Ismach, A.F. Progress, challenges, and opportunities in two-dimensional materials beyond graphene. ACS Nano 2013, 7, 2898-2926. [CrossRef]

8. Ren, K.; Sun, M.; Luo, Y.; Wang, S.; Yu, J.; Tang, W. First-principle study of electronic and optical properties of two-dimensional materials-based heterostructures based on transition metal dichalcogenides and boron phosphide. Appl. Surf. Sci. 2019, 476, 70-75. [CrossRef]

9. Zhang, H.; Chhowalla, M.; Liu, Z. 2d nanomaterials: Graphene and transition metal dichalcogenides. Chem. Soc. Rev. 2018, 47, 3015-3017. [CrossRef]

10. Radisavljevic, B.; Radenovic, A.; Brivio, J.; Giacometti, V.; Kis, A. Single-layer $\mathrm{MoS}_{2}$ transistors. Nat. Nanotechnol. 2011, 6, 147-150. [CrossRef]

11. Kaplan, D.; Gong, Y.; Mills, K.; Swaminathan, V.; Ajayan, P.M.; Shirodkar, S.; Kaxiras, E. Excitation intensity dependence of photoluminescence from monolayers of $\mathrm{MoS}_{2}$ and $\mathrm{WS}_{2} / \mathrm{MoS}_{2}$ heterostructures. 2D Mater. 2016, 3, 015005. [CrossRef]

12. Ren, K.; Wang, K.; Cheng, Y.; Tang, W.; Zhang, G. Two-dimensional heterostructures for photocatalytic water splitting: A review of recent progress. Nano Futures 2020, 4, 032006. [CrossRef]

13. Mak, K.F.; Lee, C.; Hone, J.; Shan, J.; Heinz, T.F. Atomically thin $\mathrm{MoS}_{2}$ : A new direct-gap semiconductor. Phys. Rev. Lett. 2010, 105, 136805. [CrossRef]

14. Hong, X.; Kim, J.; Shi, S.F.; Zhang, Y.; Jin, C.; Sun, Y.; Tongay, S.; Wu, J.; Zhang, Y.; Wang, F. Ultrafast charge transfer in atomically thin $\mathrm{MoS}_{2} / \mathrm{WS}_{2}$ heterostructures. Nat. Nanotechnol. 2014, 9, 682-686. [CrossRef]

15. Sun, M.; Schwingenschlögl, U. Structure prototype outperforming mxenes in stability and performance in metal-ion batteries: A high throughput study. Adv. Energy Mater. 2021, 11, 2003633. [CrossRef]

16. Sun, M.; Yan, Y.; Schwingenschlögl, U. Beryllene: A promising anode material for Na-and K-ion batteries with ultrafast charge/discharge and high specific capacity. J. Phys. Chem. Lett. 2020, 11, 9051-9056. [CrossRef]

17. Sun, M.; Schwingenschlögl, U. $\mathrm{B}_{2} \mathrm{P}_{6}$ : A two-dimensional anisotropic janus material with potential in photocatalytic water splitting and metal-ion batteries. Chem. Mater. 2020, 32, 4795-4800. [CrossRef]

18. Novoselov, K.S.; Mishchenko, A.; Carvalho, A.; Castro Neto, A.H. 2D materials and van der waals heterostructures. Science 2016, 353, aac9439. [CrossRef] [PubMed]

19. Duong, D.L.; Yun, S.J.; Lee, Y.H. Van der waals layered materials: Opportunities and challenges. ACS Nano 2017, 11, 11803-11830. [CrossRef] [PubMed]

20. Liu, Y.; Weiss, N.O.; Duan, X.; Cheng, H.-C.; Huang, Y.; Duan, X. Van der waals heterostructures and devices. Nat. Rev. Mater. 2016, 1, 16042. [CrossRef]

21. Ren, K.; Wang, S.; Luo, Y.; Chou, J.-P.; Yu, J.; Tang, W.; Sun, M. High-efficiency photocatalyst for water splitting: A janus MoSSe $/ \mathrm{XN}(\mathrm{X}=\mathrm{Ga}, \mathrm{Al})$ van der waals heterostructure. J. Phys. Phys. D Appl. Phys. 2020, 53, 185504. [CrossRef]

22. Li, J.; Huang, Z.; Ke, W.; Yu, J.; Ren, K.; Dong, Z. High solar-to-hydrogen efficiency in Arsenene/GaX (X= S, Se) van der waals heterostructure for photocatalytic water splitting. J. Alloys Compd. 2021, 866, 158774. [CrossRef]

23. Ren, K.; Luo, Y.; Wang, S.; Chou, J.-P.; Yu, J.; Tang, W.; Sun, M. A van der waals heterostructure based on graphene-like gallium nitride and boron selenide: A high-efficiency photocatalyst for water splitting. ACS Omega 2019, 4, 21689-21697. [CrossRef] [PubMed]

24. Ren, K.; Yu, J.; Tang, W. Two-dimensional ZnO/BSe van der waals heterostructure used as a promising photocatalyst for water splitting: A dft study. J. Alloys Compd. 2020, 812, 152049. [CrossRef]

25. Ren, K.; Yu, J.; Tang, W. A two-dimensional vertical van der waals heterostructure based on g-Gan and $\mathrm{Mg}(\mathrm{OH})_{2}$ used as a promising photocatalyst for water splitting: A first-principles calculation. J. Appl. Phys. 2019, 126, 065701. [CrossRef] 
26. Luo, Y.; Wang, S.; Shu, H.; Chou, J.-P.; Ren, K.; Yu, J.; Sun, M. A MoSSe/blue phosphorene vdw heterostructure with energy conversion efficiency of $19.9 \%$ for photocatalytic water splitting. Semicond. Sci. Technol. 2020, 35, 125008. [CrossRef]

27. Lee, C.H.; Lee, G.H.; van der Zande, A.M.; Chen, W.; Li, Y.; Han, M.; Cui, X.; Arefe, G.; Nuckolls, C.; Heinz, T.F.; et al. Atomically thin p-n junctions with van der waals heterointerfaces. Nat. Nanotechnol. 2014, 9, 676-681. [CrossRef]

28. Meng, R.; Jiang, J.; Liang, Q.; Yang, Q.; Tan, C.; Sun, X.; Chen, X. Design of graphene-like gallium nitride and WS ${ }_{2} / W_{S e}$ nanocomposites for photocatalyst applications. Sci. China. Mater. 2016, 59, 1027-1036. [CrossRef]

29. Roy, T.; Tosun, M.; Kang, J.S.; Sachid, A.B.; Desai, S.B.; Hettick, M.; Hu, C.C.; Javey, A. Field-effect transistors built from all two-dimensional material components. ACS Nano 2014, 8, 6259-6264. [CrossRef]

30. Long, M.; Liu, E.; Wang, P.; Gao, A.; Xia, H.; Luo, W.; Wang, B.; Zeng, J.; Fu, Y.; Xu, K.; et al. Broadband photovoltaic detectors based on an atomically thin heterostructure. Nano Lett. 2016, 16, 2254-2259. [CrossRef]

31. Ren, K.; Luo, Y.; Yu, J.; Tang, W. Theoretical prediction of two-dimensional ZnO/GaN van der waals heterostructure as a photocatalyst for water splitting. Chem. Phys. 2020, 528, 110539. [CrossRef]

32. Sun, M.; Chou, J.-P.; Ren, Q.; Zhao, Y.; Yu, J.; Tang, W. Tunable schottky barrier in van der waals heterostructures of graphene and g-GaN. Appl. Phys. Lett. 2017, 110, 173105. [CrossRef]

33. Sun, M.; Chou, J.-P.; Yu, J.; Tang, W. Effects of structural imperfection on the electronic properties of graphene/WSe 2 heterostructures. J. Mater. Chem. C 2017, 5, 10383-10390. [CrossRef]

34. Lu, A.Y.; Zhu, H.; Xiao, J.; Chuu, C.P.; Han, Y.; Chiu, M.H.; Cheng, C.C.; Yang, C.W.; Wei, K.H.; Yang, Y.; et al. Janus monolayers of transition metal dichalcogenides. Nat. Nanotechnol. 2017, 12, 744-749. [CrossRef] [PubMed]

35. Zhang, J.; Jia, S.; Kholmanov, I.; Dong, L.; Er, D.; Chen, W.; Guo, H.; Jin, Z.; Shenoy, V.B.; Shi, L.; et al. Janus monolayer transition-metal dichalcogenides. ACS Nano 2017, 11, 8192-8198. [CrossRef] [PubMed]

36. Wang, J.; Shu, H.; Zhao, T.; Liang, P.; Wang, N.; Cao, D.; Chen, X. Intriguing electronic and optical properties of two-dimensional janus transition metal dichalcogenides. Phys. Chem. Chem. Phys. 2018, 20, 18571-18578. [CrossRef] [PubMed]

37. Li, F.; Wei, W.; Zhao, P.; Huang, B.; Dai, Y. Electronic and optical properties of pristine and vertical and lateral heterostructures of Janus MoSSe and WSSe. J. Phys. Chem. Lett. 2017, 8, 5959-5965. [CrossRef]

38. Demirci, S.; Avazl1, N.; Durgun, E.; Cahangirov, S. Structural and electronic properties of monolayer group III monochalcogenides. Phys. Rev. B 2017, 95, 115409. [CrossRef]

39. Wang, B.-J.; Li, X.-H.; Zhao, R.; Cai, X.-L.; Yu, W.-Y.; Li, W.-B.; Liu, Z.-S.; Zhang, L.-W.; Ke, S.-H. Electronic structures and enhanced photocatalytic properties of blue phosphorene/BSe van der waals heterostructures. J. Mater. Chem. A 2018, 6, 8923-8929. [CrossRef]

40. Capelle, K. A bird's-eye view of density-functional theory. Braz. J. Phys. 2006, 36, 1318-1343. [CrossRef]

41. Grimme, S.; Antony, J.; Ehrlich, S.; Krieg, H. A consistent and accurate ab initio parametrization of density functional dispersion correction (DFT-D) for the 94 elements H-Pu. J. Chem. Phys. 2010, 132, 154104. [CrossRef]

42. Perdew, J.P.; Burke, K.; Ernzerhof, M. Generalized gradient approximation made simple. Phys. Rev. Lett. 1996, 77, 3865. [CrossRef]

43. Kresse, G.; Furthmüller, J. Efficiency of ab-initio total energy calculations for metals and semiconductors using a plane-wave basis set. Comp. Mater. Sci. 1996, 6, 15-50. [CrossRef]

44. Kresse, G.; Furthmüller, J. Efficient iterative schemes for ab initio total-energy calculations using a plane-wave basis set. Phys. Rev. B 1996, 54, 11169. [CrossRef] [PubMed]

45. Kresse, G.; Joubert, D. From ultrasoft pseudopotentials to the projector augmented-wave method. Phys. Rev. B 1999, 59, 1758. [CrossRef]

46. Ren, K.; Ren, C.; Luo, Y.; Xu, Y.; Yu, J.; Tang, W.; Sun, M. Using van der waals heterostructures based on two-dimensional blue phosphorus and $\mathrm{XC}(\mathrm{X}=\mathrm{Ge}, \mathrm{Si})$ for water-splitting photocatalysis: A first-principles study. Phys. Chem. Chem. Phys. 2019, 21, 9949-9956. [CrossRef] [PubMed]

47. Ren, K.; Yu, J.; Tang, W. First-principles study of two-dimensional van der waals heterostructure based on $\mathrm{ZnO}$ and $\mathrm{Mg}(\mathrm{OH})_{2}: \mathrm{A}$ potential photocatalyst for water splitting. Phys. Lett. A 2019, 383, 125916. [CrossRef]

48. Hu, T.; Jia, F.; Zhao, G.; Wu, J.; Stroppa, A.; Ren, W. Intrinsic and anisotropic rashba spin splitting in janus transition-metal dichalcogenide monolayers. Phys. Rev. B 2018, 97, 235404. [CrossRef]

49. Zhang, D.; Zhou, Z.; Hu, Y.; Yang, Z. WS 2 /BSe van der waals type-II heterostructure as a promising water splitting photocatalyst. Mater. Res. Express 2018, 6, 035513. [CrossRef]

50. Guo, W.; Ge, X.; Sun, S.; Xie, Y.; Ye, X. The strain effect on the electronic properties of the MoSSe/WSSe van der waals heterostructure: A first-principles study. Phys. Chem. Chem. Phys. 2020, 22, 4946-4956. [CrossRef] [PubMed]

51. Luo, Y.; Ren, K.; Wang, S.; Chou, J.-P.; Yu, J.; Sun, Z.; Sun, M. First-principles study on transition-metal dichalcogenide/BSe van der waals heterostructures: A promising water-splitting photocatalyst. J. Phys. Chem. C 2019, 123, 22742-22751. [CrossRef]

52. Chen, X.; Tian, F.; Persson, C.; Duan, W.; Chen, N.X. Interlayer interactions in graphites. Sci. Rep. 2013, 3, 3046. [CrossRef] [PubMed]

53. Nosé, S. A unified formulation of the constant temperature molecular dynamics methods. J. Chem. Phys. 1984, 81, 511-519. [CrossRef]

54. Wang, B.-J.; Li, X.-H.; Cai, X.-L.; Yu, W.-Y.; Zhang, L.-W.; Zhao, R.-Q.; Ke, S.-H. Blue phosphorus $/ \mathrm{Mg}(\mathrm{OH})_{2}$ van der waals heterostructures as promising visible-light photocatalysts for water splitting. J. Phys. Chem. C 2018, 122, 7075-7080. [CrossRef] 
55. Zhuang, H.L.; Hennig, R.G. Single-layer group-iii monochalcogenide photocatalysts for water splitting. Chem. Mater. 2013, 25, 3232-3238. [CrossRef]

56. Henkelman, G.; Arnaldsson, A.; Jónsson, H. A fast and robust algorithm for bader decomposition of charge density. Comp. Mater. Sci. 2006, 36, 354-360. [CrossRef]

57. Sanville, E.; Kenny, S.D.; Smith, R.; Henkelman, G. Improved grid-based algorithm for bader charge allocation. J. Comput. Chem. 2007, 28, 899-908. [CrossRef] [PubMed]

58. Fu, C.F.; Sun, J.; Luo, Q.; Li, X.; Hu, W.; Yang, J. Intrinsic electric fields in two-dimensional materials boost the solar-to-hydrogen efficiency for photocatalytic water splitting. Nano Lett. 2018, 18, 6312-6317. [CrossRef] [PubMed]

59. Ren, K.; Tang, W.; Sun, M.; Cai, Y.; Cheng, Y.; Zhang, G. A direct Z-scheme $\mathrm{PtS}_{2}$ / Arsenene van der waals heterostructure with high photocatalytic water splitting efficiency. Nanoscale 2020, 12, 17281-17289. [CrossRef] [PubMed] 